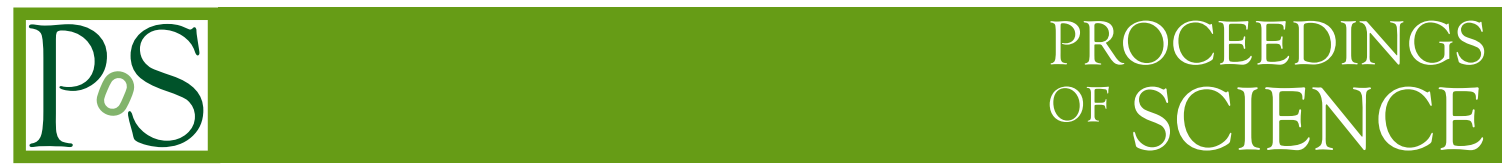

\title{
Higher Curvature Supergravity
}

\section{Dalianis, A. Kehagias* and I. Taskas}

Physics Division, National Technical University of Athens

15780 Zografou Campus, Athens, Greece

We discuss higher curvature (super) gravity in four dimensions. We describe the spectrum of the theory which contains a massive spin-two ghost and we propose a way to turn it into a canonical massive spin-two field at the linearized level.

Corfu Summer Institute 2019 "School and Workshops on Elementary Particle Physics and Gravity" (CORFU2019)

31 August - 25 September 2019

Corfu, Greece

${ }^{*}$ Speaker. 
Higher curvature theories are theories of the general form $R+\mathscr{R}^{n}$, where $R$ is the Ricci scalar describing Einstein gravity and $\mathscr{R}^{n}$ denotes $n^{\text {th }}$ power of the Weyl, Riemann and Ricci tensors and the curvature scalar [1, 2, 3, 4, 5, 6, 7, 8]. This class of models has also been explored in [9]. In particular, as has been noted in [1], the inclusion of quadratic curvature terms in the action makes the theory renormalizable. But there is a price to be paid namely the appearance of a massive ghost states. This ghost state originates from the square of the Riemann, the Ricci or the Weyl tensor. A particular class of such models, the $R+R^{2}$ supergravity has been studied [10] and it is connected to the inflationary dynamics of the Starobinsky model [11]. This models propagates the usual massless graviton and a massive spin- 0 state, the "scalaron". The latter can be identified with the inflaton field. Therefore, in the $R+R^{2}$ theory, inflation is driven entirely by the gravity itself, without the need for an extra scalar and thus making the theory quite appealing. In addition, the theory can easily be embedded in $\mathscr{N}=1$ supergravity, and its spectrum in has been determined in [13] and [14].

However, the $R+R^{2}$ theory should also include only the square of the Weyl tensor which is also quadratic in the curvature since terms quadratic in the Riemann (or Ricci) tensor are related to the square of the Weyl or the scalar curvature by the 4D Gauss-Bonnet topological term. The inclusion of the Weyl tensor now leas to a considerable problem: the theory now propagates a ghost spin-2 massive state [1]. It should be noted here that such states leads to the impossibility of maintain at one shot both unitarity and propagation of positive energy states. Indeed, using a $+i \varepsilon$ choice, opposite to the stanfdard one, one may achieve unitarity but in this case, negative energies are propagaiting forward in time.

Here we will describe the massive Weyl ${ }^{2}$ theory, which contains also the standard EinsteinHilbert action, and its supersymmetric extensions. The latter are the $\mathscr{N}$-extended Weyl ${ }^{2}$ supergravities. The inclusion of the Einstein-Hilbert action can be regarded as a mass deformation of the massless theory. In addition, the maximal $\mathscr{N}=8$ of Poincare supergravity corresponds to $\mathscr{N}=4$ Weyl supergravity [15].

An interesting limit is the massless limit $m \rightarrow 0$ of the $m^{2} R+$ Weyl $^{2}$ theory $[16,17,18]$ of the conformal supergravity $[19,20,21]$. These theories are very interesting although they propagate ghost sates as in the massless limits, i.e. no Einstein-Hilbert term, they are simple examples of superconformal theories with four derivative terms.

Let us now discuss the linearized super-Weyl ${ }^{2}$ theory. The super-Weyl tensor $\mathscr{W}_{\alpha \beta \gamma}$ is a chiral superfield, where $\alpha, \beta, \gamma=1,2$ are standard $S L(2, \mathbf{C})$ spinor indices. The spin of the highest component of the Weyl superfield $\mathscr{W}_{\alpha \beta \gamma}$ is a fermionic spin-3/2 field and therefore, the spin of the Weyl multiplet is is $\left(\frac{3}{2}, 0\right)$. It also contains the spin-two field $g_{\mu \nu}$ with five degrees of freedom and it satisfies

$$
\mathscr{W}_{\alpha \beta \gamma}=\mathscr{W}_{(\alpha \beta \gamma)},\left(\mathscr{W}_{\alpha \beta \gamma}\right)^{*}=\overline{\mathscr{W}}_{\dot{\alpha} \dot{\beta} \dot{\gamma}}, \overline{\mathscr{D}}_{\dot{\delta}} \mathscr{W}_{\alpha \beta \gamma}=0,
$$

Its bosonic content follows from

$$
\mathscr{D}_{\gamma} \mathscr{W}_{\delta \varepsilon \alpha} \mid=\frac{1}{6}\left(\frac{i}{4} \varepsilon_{\gamma \delta} \mathscr{D}_{\varepsilon \dot{\varepsilon}} A_{\alpha}^{\dot{\varepsilon}}-\frac{1}{4} \varepsilon_{\varepsilon}^{\beta} \varepsilon_{\gamma}^{\rho} W_{\delta \beta \alpha \rho}\right)+(\delta \varepsilon \alpha)
$$

where $W_{\delta \beta \alpha \rho}$ and $A_{\alpha}^{\dot{\varepsilon}}$ are the Weyl tensor and the vector auxiliary of the $\mathscr{N}=1$ supergravity (in spinorial notation). $(\delta \varepsilon \alpha)$ denotes the additional five terms from the symmetrization of the fermionic indices $\delta, \varepsilon$ and $\alpha$ [31]. 
It turns out that the supersymmetric action of the massive Weyl gravity is

$$
\mathscr{L}_{\Phi, \mathscr{W}}=\int d^{2} \Theta 2 \mathscr{E} \mathscr{R}+4 \int d^{2} \Theta 2 \mathscr{E} \tau \mathscr{W}^{2}+\text { c.c. }
$$

where we have denoted as $\tau$ the complex coupling

$$
\tau=\frac{1}{g^{2}}+i \alpha
$$

The bosonic sector of (3) turns out to be

$e^{-1} \mathscr{L}_{\mathscr{W}}=\frac{1}{2} R+\frac{1}{3} A_{\mu} A^{\mu}-\frac{1}{3} u \bar{u}+\frac{\alpha}{2}\left(\frac{1}{2} R_{H P}^{2}-\frac{2}{3} F_{\mu \nu} F_{\rho \sigma} \varepsilon^{\mu \nu \rho \sigma}\right)+\frac{1}{2 g^{2}}\left(W^{\mu \nu \rho \sigma} W_{\mu v \rho \sigma}-\frac{4}{3} F^{\mu v} F_{\mu \nu}\right)$,

where $R_{H P}^{2}=W_{\mu \nu \kappa \lambda} \varepsilon^{\kappa \lambda \rho \sigma} W_{\rho \sigma}^{\mu v}$ is the topological Hirzebruch-Pontryagin term, $F_{\mu v}=\partial_{\mu} A_{v}-$ $\partial_{v} A_{\mu}$ is the field strength of the "auxiliary" $A_{\mu}$ and $u$ is the auxiliary scalar. Note however, that the vector "auxiliary" $A_{\mu}$ is dynamical in Weyl supergravity.

It is now straightforward to determine the spectrum of the massive $\mathscr{N}=1$ Weyl supergravity. It contains the following states:

(i) The standard massless spin-2 graviton multiplet $\left(h, q_{R}\right)$ with helicity $h$ and $q_{R} U(1)_{R}$ charges, with $n_{B}+n_{F}=4$ dof:

$$
g_{\mathscr{N}=1}:(+2,0)+\left(+\frac{3}{2},+\frac{1}{2}\right)
$$

together with its CPT conjugate

$$
\left(-\frac{3}{2},-\frac{1}{2}\right)+(-2,0)
$$

(ii) A massive spin-two supermultiplet in the non-standard sector. This is the massive $\mathscr{N}=1$ super-Weyl multiplet [33] with $n_{B}+n_{F}=16$ dof:

$$
w_{\mathscr{N}=1}: \operatorname{Spin}(2)+\underline{2} \times \operatorname{Spin}(3 / 2)+\operatorname{Spin}(1)
$$

The massive states in $\mathscr{N}=1$ supergravity are arranged in $U S p(2)$ representations.

Therefore, the massive super-(Weyl $)^{2}$ gravity theory contains a total of $n_{B}+n_{F}=20$ dof.

Similarly, the spectrum of the all existing $\mathscr{N}$-extended $\mathrm{Weyl}^{2}$ supergravities $(\mathscr{N}=1,2,3,4)$ in $4 \mathrm{D}$ can be determined. The physical spectrum of the various Weyl-supergravity theories is summarized in the following table. The top helicities of each multiplet together with its CPT conjugate are denoted in the first column [16, 17]: 


\begin{tabular}{|c||c|c|c|c|}
\hline & $\mathscr{N}=4$ & $\mathscr{N}=3$ & $\mathscr{N}=2$ & $\mathscr{N}=1$ \\
\hline \hline$h_{\max }=2$ & 2 & & & \\
$h_{\max }^{\mathrm{CPT}}=0$ & 2 & & & \\
\hline$h_{\max }=3 / 2$ & 4 & & & \\
$h_{\max }^{\mathrm{CPT}}=1 / 2$ & 4 & & & \\
\hline \hline$h_{\max }=2$ & & 2 & & \\
$h_{\max }^{\mathrm{CPT}}=-1 / 2$ & & 2 & & \\
\hline$h_{\max }=3 / 2$ & & 3 & & \\
$h_{\max }^{\mathrm{CPT}}=0$ & & 3 & & \\
\hline$h_{\max }=1$ & & 1 & & \\
$h_{\max }^{\mathrm{CPT}}=1 / 2$ & & 1 & & \\
\hline \hline$h_{\max }=2$ & & & 2 & \\
$h_{\max }^{\mathrm{CPT}}=-1$ & & & 2 & \\
\hline$h_{\max }=3 / 2$ & & & 2 & \\
$h_{\max }^{\mathrm{CPT}}=-1 / 2$ & & & 2 & \\
\hline$h_{\max }=1$ & & & 1 & \\
$h_{\max }^{\mathrm{CPT}}=0$ & & & 1 & \\
\hline \hline$h_{\max }=2$ & & & & 2 \\
$h_{\max }^{\mathrm{CPT}}=-3 / 2$ & & & & 2 \\
\hline$h_{\max }=3 / 2$ & & & & 1 \\
$h_{\max }^{\mathrm{CPT}}=-1$ & & & & 1 \\
\hline$h_{\max }=1$ & & & & 1 \\
$h_{\max }^{\mathrm{CPT}}=-1 / 2$ & & & & 1 \\
\hline
\end{tabular}
action

The bosonic massive Weyl ${ }^{2}$ gravity theory in four dimensions is described by has the following

$$
S=\int d^{4} x \sqrt{-g}\left(\frac{a}{2} W_{\mu \nu \rho \sigma} W^{\mu v \rho \sigma}+\kappa^{2} R\right)
$$

where $W_{\mu v \rho \sigma}=R_{\mu v \rho \sigma}-g_{\mu[\sigma} R_{\rho] v}-g_{\mu[\rho} R_{\sigma] v}-R / 3 g_{\mu[\rho} g_{\sigma] v}$ is the Weyl tensor. The Weyl ${ }^{2}$-term is conformal invariant since under the conformal transformation

$$
g_{\mu v} \rightarrow \widehat{g}_{\mu v}=\Omega^{2} g_{\mu v}
$$

the Weyl tensor is inert

$$
\widehat{W}_{v \rho \sigma}^{\mu}=W_{v \rho \sigma}^{\mu} .
$$

Contrary, the Einstein-Hilbert term transforms under conformal transformations, as

$$
\widehat{R}=\Omega^{-2} R-6 \Omega^{-3} g^{\mu \nu} \nabla_{\mu} \nabla_{\nu} \Omega
$$

and thus, it can be regarded as a mass term in the theory.

The eom can be written as

$$
B_{\mu v}+\frac{2 \kappa^{2}}{a} G_{\mu v}=0,
$$


where $G_{\mu \nu}$ is the Einstein tensor and the Bach tensor $B_{\mu \nu}$ is given by

$$
B_{\mu \nu}=\nabla^{\rho} \nabla_{\sigma} W_{\mu \rho v}^{\sigma}+\frac{1}{2} R^{\rho \sigma} W_{\rho \mu \sigma v} .
$$

The Bach tensor transforms covarinatly under conformal transformations

$$
\widehat{B}_{\mu \nu}=\Omega^{-2} B_{\mu \nu} .
$$

It is also symmetric and traceless due to conformal invariance as well as divergence-free due to diff-invariance

$$
B_{\mu}^{\mu}=0, \quad \nabla^{\mu} B_{\mu v}=0 .
$$

To find the spectrum of the theory one should determine the poles in the propagators. It turns out that we have [1]

(i) A helicity- \pm 2 massless standard graviton $g_{\mu \nu}$.

(ii) A massive spin-two particle $w_{\mu \nu}$ with mass $\kappa^{2} / a$. It is due to the Weyl $\mathrm{l}^{2}$ term and it is is a ghost for $a>0$ or a tachyon for $a<0$. This is the non-standard sector of the theory.

Therefore, the massive (Weyl) ${ }^{2}$ gravity theory contains seven propagating dof.

An alternative way to identify the spectrum of the bosonic massive Weyl theory is to express it as a bimetric gravity theory with two spin- 2 fields $g_{\mu \nu}$ and $w_{\mu \nu}$. Consider for example the action

$$
S=\int_{\mathscr{M}} d^{4} x \sqrt{-g}\left(M_{P}^{2} R+c_{1} W_{\mu v \rho \sigma} W^{\mu v \rho \sigma}+c_{2} R^{2}\right) .
$$

Now, let us introduce a second symmetric tensor field $w_{\mu \nu}$. It can be shown that the two-derivative action

$$
S=\int_{\mathscr{M}} d^{4} x \sqrt{-g}\left(M_{P}^{2} R(g)+\frac{2}{g_{W}} G_{\mu v}(g) w^{\mu v}-\left(w^{\mu v} w_{\mu v}-a w^{2}\right)\right) .
$$

where $G_{\mu \nu}=R_{\mu \nu}-1 / 2 R g_{\mu \nu}$ is the Einstein tensor for the metric $g_{\mu \nu}$ and the last term describes a mass term for $w_{\mu \nu}$ is classically equivalent to (18). Indeed, using the equation of motion for $w_{\mu \nu}$

$$
\frac{\delta S}{\delta w_{\mu \nu}} \Rightarrow w_{\mu v}=\frac{1}{g_{W}}\left(R_{\mu v}(g)-\frac{1}{6} g_{\mu v} R\right)
$$

in the action (19), it turns out that the theory is classically equivalent to (18) since

$$
W_{\mu v \rho \sigma} W^{\mu v \rho \sigma}=G B+2\left(R_{\mu v} R^{\mu v}-\frac{1}{3} R^{2}\right),
$$

where $G B$ is the Gauss-Bonnet term. In fact we find that

$$
c_{1}=\frac{1}{2 g_{W}^{2}}, \quad c_{2}=\frac{a-1}{4 a-1} \frac{1}{3 g_{W}^{2}} .
$$

Therefore, for $a=1$ the scalar mode associated to the $R^{2}$ term is absent and in this case, (19) describes massive Weyl gravity. 
Eq.(19) describes a massless spin-2 $g_{\mu \nu}$ together with massive spin-2 $w_{\mu \nu}$ (for $\alpha=1$ ). Note that the kinetic term for the massive spin-2 $w_{\mu \nu}$ is implicit in the in mixed term $G_{\mu v}(g) w^{\mu \nu}$. In order to explicitly see this, let us consider fluctuations $h_{\mu \nu}=g_{\mu v}-\eta_{\mu v}$ around Minkowski spacetime (metric $\eta_{\mu v}$ ). Then we find that to quadratic order we have

$$
\mathscr{S}_{2}=\int d^{4} x \sqrt{-g}\left\{-\frac{M_{P}^{2}}{2} h^{\mu v} \mathscr{E}_{\mu \nu}^{\alpha \beta} h_{\alpha \beta}+\frac{1}{g_{W}} w^{\mu v} \mathscr{E}_{\mu \nu}^{\alpha \beta} h_{\alpha \beta}-\left(w^{\mu v} w_{\mu v}-w^{2}\right)\right\}
$$

where $g_{W}=M_{W} / M_{P}$ and $\mathscr{E}_{\mu \nu}^{\alpha \beta}$ is as usual the tensor

$$
\mathscr{E}_{\mu v}^{\alpha \beta} h_{\alpha \beta}=-\frac{1}{2}\left\{\square h_{\mu v}-2 \partial_{(\mu} \partial_{\alpha} h_{v)}^{\alpha}+\partial_{\mu} \partial_{v} h-\eta_{\mu v}\left(\square h-\partial_{\alpha} \partial_{\beta} h^{\alpha \beta}\right)\right\} .
$$

Let us now define a new field

$$
\bar{h}_{\mu v}=h_{\mu v}-\frac{2}{g_{W} M_{P}^{2}} w_{\mu \nu},
$$

in tersm of which, the quadratic action (23) turns out to be written as

$$
\mathscr{S}_{2}=\int d^{4} x \sqrt{-g}\left\{-\frac{M_{P}^{2}}{2} \bar{h}^{\mu v} \mathscr{E}_{\mu v}^{\alpha \beta} \bar{h}_{\alpha \beta}+\frac{1}{g_{W}^{2} M_{P}^{2}} w^{\mu v} \mathscr{E}_{\mu v}^{\alpha \beta} w_{\alpha \beta}-\left(w^{\mu v} w_{\mu v}-w^{2}\right)\right\}
$$

The field $\bar{h}_{\mu \nu}$ is clearly the massless graviton and $w_{\mu \nu}$ is a second massive spin-2 tensor field. Note however that the wrong sign (compared to $\bar{h}_{\mu v}$ ) of the kinetic term of $w_{\mu \nu}$ indicating that the second spin-2 tensor mode is actually a ghost. The bimetric gravity theory (19) may be turned into a ghost-free theory by the inclusion of an infinite number of terms. This procedure has been shown in [34] to be equivalent to adding an infinite number of higher derivative terms.

Another way to remove the ghost stae is the following. Let us add to the action (19) a term of the form

$$
c w^{\mu v} \mathscr{E}_{\mu \nu} w_{a b}
$$

with

$$
c<-\frac{2}{g_{w}^{2} M_{P}^{2}}
$$

This term clearly removes the ghost, and, thus the theory will contain a graviton and a massive spin-2 particle with canonical kinetic term. The action in this case reads

$$
S=\int d^{4} x \sqrt{-g}\left(\mathrm{M}_{P}^{2} R+\frac{2}{g_{w}} G_{\mu v} w^{\mu v}-\left(w^{\mu v} w_{\mu v}-w^{2}\right)+c w^{\mu v} \mathscr{E}_{\mu v}^{a b} w_{a b}\right)
$$

Notice that the derivatives and the metric that appear in the definition of $\mathscr{E}$ are now covariant derivatives and the full metric $g_{\mu \nu}$ in the previous action. The new EOM read

$$
\frac{1}{g_{w}} G_{\mu v}-w_{\mu v}+w g_{\mu v}-\frac{c}{2}\left[\square w_{\mu v}-2 \nabla_{(\mu} \nabla^{a} w_{v) a}+\nabla_{\mu} \nabla_{v} w-g_{\mu v}\left(\square w-\nabla_{a} \nabla_{b} w^{a b}\right)\right]=0
$$

or

$\frac{1}{g_{w}} G_{\mu \nu}=\left[\delta_{\mu}^{a} \delta_{v}^{b}-g_{\mu \nu} g^{a b}+\frac{c}{2}\left(\square \delta_{\mu}^{a} \delta_{v}^{b}-\nabla_{\mu} \nabla^{a} \delta_{v}^{b}-\nabla_{v} \nabla^{a} \delta_{\mu}^{b}+\nabla_{\mu} \nabla_{v} g^{a b}-g_{\mu \nu} g^{a b} \square+g_{\mu \nu} \nabla^{a} \nabla^{b}\right)\right] w_{a b}$ 
and

$$
\begin{aligned}
M_{P}^{2} G_{\mu v} & =\frac{1}{g_{w}}\left[\square w_{\mu v}-\nabla_{\sigma} \nabla_{\mu} w_{v}^{\sigma}-\nabla_{\sigma} \nabla_{v} w_{\mu}^{\sigma}+R_{\mu v} w-R w_{\mu v}+\nabla_{(\mu} \nabla_{v)} w\right. \\
& \left.-2\left(G_{\mu}^{a} w_{v a}+G_{v}^{a} w_{\mu a}\right)+g_{\mu v}\left(G_{a b} w^{a b}-\frac{g_{w}}{2}\left(w^{a b} w_{a b}-w^{2}\right)+\nabla_{a} \nabla_{b} w^{a b}-\square w\right)\right] \\
& +2\left(w_{a(\mu} w_{v)}^{a}-w w_{\mu v}\right)-2 c w_{\kappa \lambda}\left[\frac{\delta}{\delta g^{\mu v}}\left(g^{\kappa \sigma} g^{\lambda \tau} \mathscr{E}_{\sigma \tau}^{a b}\right)\right] w_{a b}+\frac{c}{2} g_{\mu v} w^{\kappa \lambda} \mathscr{E}_{\kappa \lambda} w_{a b}
\end{aligned}
$$

Flat space-time $g_{\mu v}=\eta_{\mu v}$ along with $w_{\mu \nu}=0$ remains a solution to (31) and (32). Linearizing (31) around this background yields

$$
\frac{1}{g_{w}} \delta G_{\mu v}=\left[\delta_{\mu}^{a} \delta_{v}^{b}-\eta_{\mu v} \eta^{a b}+\frac{c}{2}\left(\square \delta_{\mu}^{a} \delta_{v}^{b}-\partial_{\mu} \partial^{a} \delta_{v}^{b}-\partial_{v} \partial^{b} \delta_{\mu}^{a}+\partial_{\mu} \partial_{v} \eta^{a b}-\eta_{\mu v} \eta^{a b} \square+\eta_{\mu v} \partial^{a} \partial^{b}\right)\right] w_{a b}
$$

with

$$
\delta G_{\mu v}=\frac{1}{2}\left(\partial_{\mu} \partial^{\lambda} h_{v \lambda}+\partial_{v} \partial^{\lambda} h_{\mu \lambda}-\square h_{\mu v}-\partial_{\mu} \partial_{v} h-\eta_{\mu v} \partial^{\kappa} \partial^{\lambda} h_{\kappa \lambda}+\eta_{\mu v} \square h\right)
$$

The linearized action is then given by

$$
S^{(2)}=\int d^{4} x\left[-\frac{M_{P}^{2}}{2} \bar{h}^{\mu v} \mathscr{E}_{\mu v} a b \bar{h}_{a b}-\frac{1}{\bar{g}_{w}^{2} M_{P}^{2}} w^{\mu v} \mathscr{E}_{\mu v}^{a b} w_{a b}-\left(w^{\mu v} w_{\mu v}-w^{2}\right)\right],
$$

where

$$
-\frac{1}{\bar{g}_{w}^{2} M_{P}^{2}}=c+\frac{2}{g_{w}^{2} M_{P}^{2}}
$$

We can decompose $\bar{h}_{\mu \nu}$ and $w_{\mu \nu}$ as

$$
\begin{aligned}
& \bar{h}_{\mu v}=\bar{h}_{\mu v}^{\perp}+\partial_{\mu} A_{v}^{\perp}+\partial_{v} A_{\mu}^{\perp}+\left(\partial_{\mu} \partial_{v}-\frac{1}{4} \eta_{\mu v} \square\right) B+\frac{1}{4} \eta_{\mu v} \bar{h} \\
& w_{\mu v}=w_{\mu v}^{\perp}+\partial_{\mu} C_{v}^{\perp}+\partial_{v} C_{\mu}^{\perp}+\left(\partial_{\mu} \partial_{v}-\frac{1}{4} \eta_{\mu v} \square\right) D+\frac{1}{4} \eta_{\mu v} w
\end{aligned}
$$

where $\bar{h}_{\mu \nu}^{\perp}$ and $w_{\mu \nu}^{\perp}$ are transverse traceless and $C_{v}^{\perp}$ is transverse. Then the action (35) becomes

$$
\begin{aligned}
S^{(2)} & =\int d^{4} x\left[\frac{M_{P}^{2}}{4}\left(\bar{h}^{\perp \mu v} \square \bar{h}_{\mu v}^{\perp}-\frac{3}{8}(\bar{h}-\square B) \square(\bar{h}-\square B)\right)\right. \\
& +\frac{1}{2 \bar{g}_{w}^{2} M_{P}^{2}}\left(w^{\perp \mu v} \square w_{\mu v}^{\perp}-\frac{3}{8}(w-\square D) \square(w-\square D)\right) \\
& \left.+2 C_{\mu}^{\perp} \square C^{\perp \mu}-\frac{3}{4}(w-\square D)(w+\square D)-w_{\mu \nu}^{\perp} w^{\perp \mu v}\right]
\end{aligned}
$$

We introduce the following scalars

$$
\phi=\bar{h}-\square B \quad, \quad S=w-\square D \quad, \quad S^{\prime}=w+\square D
$$

so that (39) turns out to be

$$
\begin{aligned}
S^{(2)} & =\int d^{4} x\left[\frac{M_{P}^{2}}{4}\left(\bar{h}^{\perp \mu v} \square \bar{h}_{\mu v}^{\perp}-\frac{3}{8} \phi \square \phi\right)\right. \\
& \left.+\frac{1}{2 \bar{g}_{w}^{2} M_{P}^{2}}\left(w^{\perp \mu v} \square w_{\mu v}^{\perp}-\frac{3}{8} S \square S\right)+2 C_{\mu}^{\perp} \square C^{\perp \mu}-\frac{3}{4} S S^{\prime}-w_{\mu \nu}^{\perp} w^{\perp \mu v}\right]
\end{aligned}
$$


There is no kinetic term for the field $S^{\prime}$ and by using its EOM

$$
S^{\prime}=-\frac{1}{2 \bar{g}_{w}^{2} M_{P}^{2}} \square S
$$

the action becomes

$S^{(2)}=\int d^{4} x\left[\frac{M_{P}^{2}}{4}\left(\bar{h}^{\perp \mu v} \square \bar{h}_{\mu v}^{\perp}-\frac{3}{8} \phi \square \phi\right)+\frac{1}{2 \bar{g}_{w}^{2} M_{P}^{2}}\left(w^{\perp \mu v} \square w_{\mu v}^{\perp}+\frac{3}{8} S \square S\right)+2 C_{\mu}^{\perp} \square C^{\perp \mu}-w_{\mu \nu}^{\perp} w^{\perp \mu v}\right]$

Note that $\phi$ is the ghost of standard General Relativity. The propagating degrees of freedom are therefore, a massless spin-2 field $\bar{h}_{\mu \nu}^{\perp}$, a massive spin-2 field $w_{\mu \nu}^{\perp}$, a massless spin-1 field $C_{\mu}^{\perp}$ and a scalar $S$.

\section{Acknowledgements:}

This research is carried out / funded in the context of the project "Generalized Theories of Gravity" (MIS 5049089) under the call for proposals "Researchers' support with an emphasis on young researchers - 2nd Cycle". The project is co-financed by Greece and the European Union (European Social Fund - ESF) by the Operational Programme Human Resources Development, Education and Lifelong Learning 2014-2020.âĂ

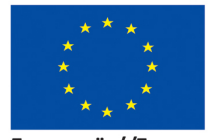

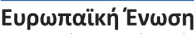

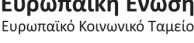

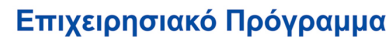

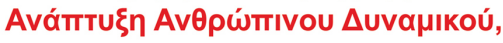

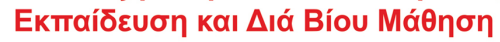

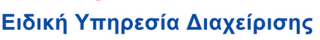

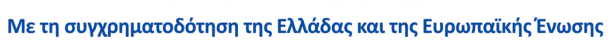

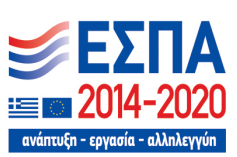

\section{References}

[1] K. S. Stelle, "Renormalization of Higher Derivative Quantum Gravity," Phys. Rev. D 16 (1977) 953. K. S. Stelle, "Classical Gravity with Higher Derivatives," Gen. Rel. Grav. 9 (1978) 353.

[2] D. G. Boulware, G. T. Horowitz and A. Strominger, "Zero Energy Theorem for Scale Invariant Gravity,” Phys. Rev. Lett. 50 (1983) 1726.

[3] F. David and A. Strominger, "On the Calculability of Newton's Constant and the Renormalizability of Scale Invariant Quantum Gravity,” Phys. Lett. B 143 (1984) 125.

[4] G. T. Horowitz, "Quantum Cosmology With a Positive Definite Action,” Phys. Rev. D 31 (1985) 1169.

[5] S. Deser and B. Tekin, "Shortcuts to high symmetry solutions in gravitational theories," Class. Quant. Grav. 20 (2003) 4877 [gr-qc/0306114]. "New energy definition for higher curvature gravities," Phys. Rev. D 75 (2007) 084032 [gr-qc/0701140].

[6] G. 't Hooft, "A class of elementary particle models without any adjustable real parameters," Found. Phys. 41 (2011) 1829 [arXiv:1104.4543 [gr-qc]].

[7] J. Maldacena, "Einstein Gravity from Conformal Gravity," arXiv:1105.5632 [hep-th].

[8] H. Lu, C. N. Pope, E. Sezgin and L. Wulff, "Critical and Non-Critical Einstein-Weyl Supergravity," JHEP 1110, 131 (2011) [arXiv:1107.2480 [hep-th]]. 
[9] L. Alvarez-Gaume, A. Kehagias, C. Kounnas, D. Lüst and A. Riotto, "Aspects of Quadratic Gravity," Fortsch. Phys. 64 (2016) no.2-3, 176 [arXiv:1505.07657 [hep-th]].

[10] S. Cecotti, "Higher Derivative Supergravity Is Equivalent To Standard Supergravity Coupled To Matter. 1.," Phys. Lett. B 190 (1987) 86.

S. Cecotti, S. Ferrara, M. Porrati and S. Sabharwal, "New Minimal Higher Derivative Supergravity Coupled To Matter,” Nucl. Phys. B 306, 160 (1988).

[11] R. Kallosh and A. Linde, "Superconformal generalizations of the Starobinsky model," JCAP 1306, 028 (2013) [arXiv:1306.3214 [hep-th]];

J. Ellis, D. V. Nanopoulos and K. A. Olive, "No-Scale Supergravity Realization of the Starobinsky Model of Inflation,” Phys. Rev. Lett. 111, 111301 (2013) Erratum: [Phys. Rev. Lett. 111, no. 12, 129902 (2013)] arXiv:1305.1247 [hep-th]; JCAP 1310, 009 (2013) [arXiv:1307.3537 [hep-th]]; F. Farakos, A. Kehagias and A. Riotto, "On the Starobinsky Model of Inflation from Supergravity," Nucl. Phys. B 876, 187 (2013) [arXiv:1307.1137 [hep-th]];

S. Ferrara, R. Kallosh, A. Linde and M. Porrati, "Minimal Supergravity Models of Inflation," Phys. Rev. D 88, no. 8, 085038 (2013) [arXiv:1307.7696 [hep-th]];

R. Kallosh, A. Linde and D. Roest, "Superconformal Inflationary $\alpha$-Attractors," JHEP 1311, 198 (2013) [arXiv:1311.0472 [hep-th]];

J. Alexandre, N. Houston and N. E. Mavromatos, "Starobinsky-type Inflation in Dynamical Supergravity Breaking Scenarios," Phys. Rev. D 89, no. 2, 027703 (2014) [arXiv:1312.5197 [gr-qc]]; S. Ferrara and M. Porrati, "Minimal $R+R^{2}$ Supergravity Models of Inflation Coupled to Matter," Phys. Lett. B 737, 135 (2014) [arXiv:1407.6164 [hep-th]];

A. Ceresole, G. Dall'Agata, S. Ferrara, M. Trigiante and A. Van Proeyen, "A search for an $\mathscr{N}=2$ inflaton potential," Fortsch. Phys. 62, 584 (2014) [arXiv:1404.1745 [hep-th]];

C. Kounnas, D. Lüst and N. Toumbas, " $\mathrm{R}^{2}$ inflation from scale invariant supergravity and anomaly free superstrings with fluxes," Fortsch. Phys. 63, 12 (2015) [arXiv:1409.7076 [hep-th]];

I. Dalianis, F. Farakos, A. Kehagias, A. Riotto and R. von Unge, "Supersymmetry Breaking and Inflation from Higher Curvature Supergravity," JHEP 1501, 043 (2015) [arXiv:1409.8299 [hep-th]]; T. Terada, Y. Watanabe, Y. Yamada and J. Yokoyama, "Reheating processes after Starobinsky inflation in old-minimal supergravity," JHEP 1502, 105 (2015) [arXiv:1411.6746 [hep-ph]];

S. Ferrara and A. Kehagias, "Higher Curvature Supergravity, Supersymmetry Breaking and Inflation," Subnucl. Ser. 52, 119 (2017) [arXiv:1407.5187 [hep-th]];

S. Ferrara, A. Kehagias and A. Sagnotti, "Cosmology and Supergravity," Int. J. Mod. Phys. A 31, no. 25, 1630044 (2016) [arXiv:1605.04791 [hep-th]].

[12] A. A. Starobinsky, "A New Type of Isotropic Cosmological Models Without Singularity," Phys. Lett. B 91, 99 (1980).

V. F. Mukhanov and G. V. Chibisov, "Quantum Fluctuation and Nonsingular Universe. (In Russian)," JETP Lett. 33, 532 (1981) [Pisma Zh. Eksp. Teor. Fiz. 33, 549 (1981)].

[13] S. Ferrara, M. T. Grisaru and P. van Nieuwenhuizen, "Poincare and Conformal Supergravity Models With Closed Algebras,” Nucl. Phys. B 138, 430 (1978).

[14] B. de Wit, J. W. van Holten and A. Van Proeyen, "Transformation Rules of N=2 Supergravity Multiplets,” Nucl. Phys. B 167 (1980) 186.

[15] B. de Wit and S. Ferrara, "On Higher Order Invariants in Extended Supergravity," Phys. Lett. 81B (1979) 317.

[16] S. Ferrara, A. Kehagias and D. LÃijst, "Aspects of Weyl Supergravity," JHEP 08 (2018), 197, arXiv:1806.10016[hep-th] 
[17] S. Ferrara, A. Kehagias and D. LÃijst, "Bimetric, Conformal Supergravity and its Superstring Embedding," JHEP 05 (2019), 100, arXiv:1810.08147 [hep-th].

[18] S. Ferrara, A. Kehagias and D. LÃijst, "Aspects of Conformal Supergravity,” arXiv:2001.04998 [hep-th].

[19] S. Ferrara, M. Kaku, P. K. Townsend and P. van Nieuwenhuizen, "Gauging the Graded Conformal Group with Unitary Internal Symmetries,” Nucl. Phys. B 129 (1977) 125.

[20] M. Kaku, P. K. Townsend and P. van Nieuwenhuizen, "Superconformal Unified Field Theory," Phys. Rev. Lett. 39 (1977) 1109.

M. Kaku, P. K. Townsend and P. van Nieuwenhuizen, "Properties of Conformal Supergravity," Phys. Rev. D 17 (1978) 3179.

[21] D. Z. Freedman and A. Van Proeyen, "Supergravity," Cambridge University Press, 2012.

[22] J. M. Cline, S. Jeon and G. D. Moore, "The Phantom menaced: Constraints on low-energy effective ghosts," Phys. Rev. D 70, 043543 (2004) [hep-ph/0311312].

[23] H. Johansson and J. Nohle, "Conformal Gravity from Gauge Theory," arXiv:1707.02965.

H. Johansson, G. Mogull and F. Teng, "Unraveling conformal gravity amplitudes," arXiv:1806.05124 [hep-th].

[24] A. Salvio, "Quadratic Gravity," arXiv:1804.09944 [hep-th].

[25] S. Ferrara and B. Zumino, "Structure of Linearized supergravity and Conformal Supergravity," Nucl. Phys. B 134 (1978) 301.

[26] R. J. Riegert, “The Particle Content Of Linearized Conformal Gravity,” Phys. Lett. A 105, 110 (1984).

[27] A. Balfagon and X. Jaen, "Review of some classical gravitational superenergy tensors using computational techniques," Class. Quant. Grav. 17, 2491 (2000) [gr-qc/9912060].

[28] L. Bel, CR Acad. Sci. Paris, 247 (1958) 1094, 248 (1959) 1297; I. Robinson, unpublished Kings College Lectures (1958); Class. Quant. Grav. 14, 4331 (1997).

[29] C. Bachas and I. Lavdas, "Massive Anti-de Sitter Gravity from String Theory," arXiv:1807.00591 [hep-th].

[30] N. Boulanger, “A Weyl-covariant tensor calculus,” J. Math. Phys. 46, 053508 (2005) [hep-th/0412314].

[31] F. Farakos, S. Ferrara, A. Kehagias and D. Lüst, "Non-linear Realizations and Higher Curvature Supergravity,” Fortsch. Phys. 65, no. 12, 1700073 (2017) [arXiv:1707.06991 [hep-th]].

[32] S. Ferrara, C. A. Savoy and B. Zumino, "General Massive Multiplets in Extended Supersymmetry," Phys. Lett. 100B (1981) 393.

[33] S. Ferrara and B. Zumino, "Transformation Properties of the Supercurrent," Nucl. Phys. B 87 (1975) 207.

S. Ferrara and B. Zumino, "Structure of Conformal Supergravity," Nucl. Phys. B 134 (1978) 301.

[34] B. Gording and A. Schmidt-May, "Ghost-free infinite derivative gravity," arXiv:1807.05011 [gr-qc]. 